\title{
Avaliação clínica de restaurações de ionômero de vidro em crianças
}

\section{Clinical evaluation of glass ionomer restorations in children}

\author{
Yêska Paola Costa Aguiar* \\ Darlene Cristina Ramos Eloy Dantas** \\ Ana Isabella Arruda Meira Ribeiro** \\ Rennaly de Freitas Lima* \\ Yasmine de Carvalho Sousa ${ }^{* * *}$ \\ Gymenna Maria Tenório Guênes ${ }^{* * * *}$
}

\section{Resumo}

O Tratamento Restaurador Atraumático (TRA) foi desenvolvido para facilitar o acesso ao serviço odontológico das populações de menor poder aquisitivo, consistindo na remoção parcial do tecido cariado, mantendo a dentina afetada, utilizando-se apenas instrumentos manuais e restaurando a cavidade com um biomaterial, cimento de ionômero de vidro (CIV). Objetivo: Avaliar clinicamente restaurações de cavidade classe I com CIV em crianças assistidas pela Fundação Sementes de Vida, Campina Grande - PB, Brasil. Materiais e método: Realizou-se um estudo intervencional, prospectivo, com método qualisquantitativo. Os exames clínicos e restaurações foram realizados em 85 infantes, os quais possuíam lesão de cárie oclusal em dentes posteriores de diâmetro maior que $3 \mathrm{~mm}$ envolvendo dentina. Após três meses, as restaurações foram avaliadas de acordo com os critérios do US Public Health Service (USPHS) modificado, sendo os resultados analisados através de técnicas estatísticas descritivas, com uso de variância oneway ANOVA e teste (T) para amostras independentes $(P<0.05)$. Resultados: Para todas as variáveis analisadas, à exceção da presença de cárie secundária, onde o escore CHARLIE (C) predominou, a avaliação apontou para resultados compatíveis com os escores ALFA (A) e BRAVO (B), o que representa significativo grau de sucesso do procedimento em foco. Conclusões: Frente à avaliação realizada, foi verificado que o TRA teve um desempenho satisfatório, em virtude da melhora do quadro clínico apresentado pelas crianças ao final do estudo.

Palavras-chave: Tratamento Dentário Restaurador Sem Trauma. Cárie Dentária. Cimentos de lonômeros de Vidro.

\section{Introdução}

A cárie dentária durante a infância é causa frequente de dor, desconforto e dificuldades de alimentação, que comprometem a qualidade de vida e o desenvolvimento biopsicossocial infantil ${ }^{1}$. Novas abordagens para o tratamento dessa patologia vêm sendo desenvolvidas com o intuito de otimizar o atendimento odontológico ${ }^{2}$. Nesse sentido, na década de 80, surgiu o Tratamento Restaurador Atraumático (TRA) ${ }^{3}$ no intuito de responder às necessidades do tratamento odontológico restaurador em lugares onde não havia nenhum equipamento para a técnica tradicional de remoção do tecido cariado com alta rotação $0^{4,5}$.

O material de eleição no TRA é o cimento de ionômero de vidro (CIV) por possuir propriedades biológicas $^{6}$. De acordo com Guideline on Pediatric Restorative Dentistry ${ }^{7}$, este têm várias características que o torna favorável para uso em crianças, tais como: ligação química tanto para esmalte quanto para dentina; expansão térmica semelhante ao da estrutura do dente; biocompatibilidade; absorção e liberação de flúor e diminuição da sensibilidade à umidade, quando comparados às resinas.

A indicação clínica para adoção do TRA, em dentes decíduos e permanentes, é a lesão cariosa em face única ${ }^{8}$. Este procedimento contribui decisivamente na elucidação da necessidade de remoção completa apenas da dentina infectada, uma vez que

\footnotetext{
Mestre em Clínica Odontológica pela Universidade Estadual da Paraíba (UEPB) - Campina Grande, PB, Brasil.

Professora Doutora do Departamento de Odontologia da Universidade Estadual da Paraíba (UEPB) - Campina Grande, PB, Brasil.

Mestranda em Odontologia (Dentística) pela Faculdade de Odontologia da Universidade de Pernambuco (FOP-UPE) - Recife, PE, Brasil.

Professora Doutora do Departamento de Odontologia da Universidade Federal de Campina Grande (UFCG) - Patos, PB, Brasil.
} 
a restauração é sempre realizada sob um tecido desmineralizado ${ }^{9}$, com um material adesivo e liberador de flúor, a fim de que ocorra a remineralização e reparo do substrato dentinário ${ }^{10}$.

Assim, este estudo avaliou clinicamente restaurações de cavidade classe I com cimento de ionômero de vidro (CIV) em crianças participantes da escola em tempo integral - Fundação Sementes de Vida na cidade de Campina Grande - PB. A hipótese nula deste trabalho foi que o tratamento restaurador atraumático não influenciaria significativamente no desempenho clínico das restaurações e no tratamento da doença cárie.

\section{Materiais e Método}

Trata-se de um estudo intervencional e prospectivo, descritivo e analítico, com método quantitativo. A técnica de pesquisa foi a observação direta intensiva por meio de exame clínico no consultório odontológico da Fundação Sementes de Vida. A realização da pesquisa ocorreu após aprovação pelo Comitê de Ética em Pesquisa da Universidade Estadual da Paraíba, Campina Grande, Paraíba, Brasil (0415.0.133.000-08).

A amostra foi constituída por 85 crianças, entre 5 e 13 anos, que possuíam lesão de cárie oclusal em dentes posteriores de diâmetro maior que $3 \mathrm{~mm}$ envolvendo dentina sem comprometimento pulpar, para as quais foi solicitado o Termo de Consentimento Livre e Esclarecido assinado pelos responsáveis.. Dentes com evidente envolvimento pulpar ou sintomatologia dolorosa e/ou presença de edema ou fístula foram excluídos do estudo. Para esses casos, a criança foi submetida a tratamento de emergência e encaminhada para posterior atendimento curativo ${ }^{11}$.
Os exames clínicos e restaurações foram realizados no consultório odontológico da Fundação por três pesquisadoras, devidamente paramentadas de acordo com as normas de controle de infecção, por meio de inspeção visual, com auxílio de luz artificial, espátula de madeira, gazes descartáveis, espelhos bucais, sondas estéreis, escavadores de dentina e espátulas de inserção, seguindo protocolo pré-estabelecido ${ }^{12}$.

A manipulação do cimento de ionômero de vidro restaurador (Vidrion $\mathrm{R}^{\circledR} \mathrm{SS}$ White, Rio de Janeiro, RJ, Brasil) foi feita de acordo com as recomendações do fabricante, utilizando uma espátula metálica Simples № 36 (Duflex ${ }^{\circledR}$ SS White, Rio de Janeiro-RJ, Brasil). O Selamento das cavidades Classe I, se deu com a inserção do material restaurador com ligeiro excesso condensado nos ângulos internos com a parte convexa do escavador de dentina. Aplicou-se vaselina sólida (Miyako do Brasil Ind Com Ltda São Paulo, SP, Brasil) sobre o dedo da luva e, com ele, exerceu-se uma leve pressão digital (técnica "finger -press”) sobre o material durante alguns segundos, para remoção dos excessos e obtenção de uma restauração com superfície mais lisa.

Com o auxílio de papel carbono articular contact dupla face (Angelus Ind. de Produtos Odontológicos S/A, Londrina, PR, Brasil), a oclusão foi checada e, quando necessário, a restauração foi desgastada com Hollemback (SS White, Rio de Janeiro, RJ, Brasil). Após estes procedimentos, os pacientes foram instruídos a não mastigar nenhum alimento sólido por, no mínimo, uma hora. As crianças, professores e responsáveis envolvidos na pesquisa receberam instruções e palestras sobre alimentação e higiene bucal $^{13}$. O estado das restaurações foi avaliado clinicamente por uma cirurgiã-dentista que não esteve envolvida na prestação do tratamento, seguindo os critérios do USPHS modificado ${ }^{14,15}$ (Tab. 1 e Fig. 1).

Tabela 1 - Critérios do USPHS modificado - Adaptado de Santiago et al ${ }^{15}(2010)$

\begin{tabular}{|c|c|c|}
\hline Categoria & Classificação & Critério \\
\hline Retenção & $\begin{array}{l}\text { Alfa } \\
\text { Bravo } \\
\text { Charlie }\end{array}$ & $\begin{array}{l}\text { A - Restauração completamente presente } \\
\text { B - Restauração parcialmente perdida, sem necessidade de troca imediata } \\
\text { C - Restauração perdida, com necessidade de troca imediata }\end{array}$ \\
\hline Integridade Marginal & $\begin{array}{l}\text { Alfa } \\
\text { Bravo } \\
\text { Charlie }\end{array}$ & $\begin{array}{l}\text { A - Sem evidência visível de fendas na margem, na qual a sonda exploradora não prende } \\
\text { B - Sonda exploradora se prende em alguma falha na margem, sem exposição de } \\
\text { dentina ou material de base } \\
\text { C - Evidência visível de uma fenda na margem da restauração, com exposição de dentina } \\
\text { ou material de base ou a restauração está solta na cavidade, fraturada, falta alguma parte } \\
\text { da restauração ou ela está ausente }\end{array}$ \\
\hline Descoloração & Alfa & A - Sem descoloração evidente da margem da restauração \\
\hline Marginal & $\begin{array}{l}\text { Bravo } \\
\text { Charlie }\end{array}$ & $\begin{array}{l}\text { B - Leve pigmentação da margem da restauração } \\
\text { C - Pigmentação evidente sem possibilidade de polimento. }\end{array}$ \\
\hline Forma Anatômica & $\begin{array}{l}\text { Alfa } \\
\text { Bravo } \\
\text { Charlie }\end{array}$ & $\begin{array}{l}\text { A - A restauração apresenta uma correta anatomia, não desgastada } \\
\text { B - A restauração está desgastada, porém sem dentina exposta } \\
\text { C - Existe perda substancial de material, deixando dentina exposta. }\end{array}$ \\
\hline Rugosidade Superficial & $\begin{array}{l}\text { Alfa } \\
\text { Bravo } \\
\text { Charlie }\end{array}$ & $\begin{array}{l}\text { A - Superfície lisa sem irritação dos tecidos adjacentes } \\
\text { B - Superfície opaca, leve rugosidade / Superfície com irregularidades superficiais } \\
\text { C - Irregularidades profundas estão presentes, a superfície está fraturada ou lascada. }\end{array}$ \\
\hline $\begin{array}{l}\text { Presença de Cárie } \\
\text { Secundária }\end{array}$ & $\begin{array}{l}\text { Alfa } \\
\text { Charlie }\end{array}$ & $\begin{array}{l}\text { A - A restauração é considerada livre de cárie } \\
\text { C - É detectada cárie secundária }\end{array}$ \\
\hline
\end{tabular}



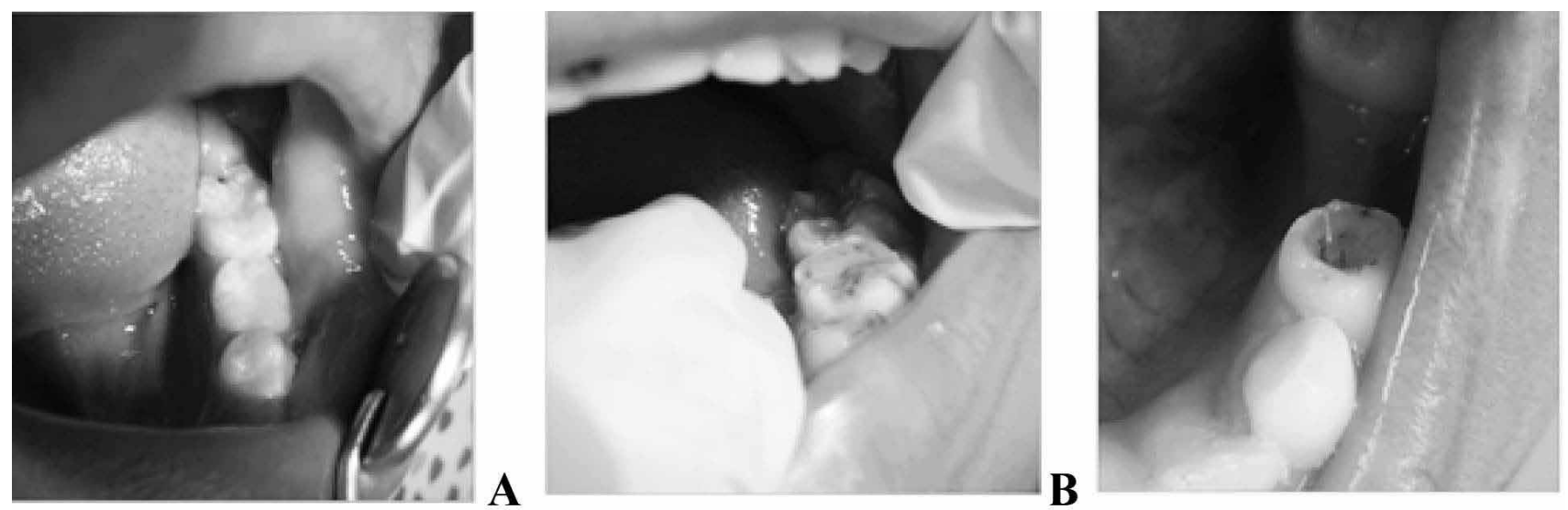

Figura 1 - Aspecto clínico das classificações ALFA, BRAVO e CHARLIE. A - Restauração "ALFA" sem alteração; B - Restauracão "BRAVO" com alteração clinicamente aceitáveis; C - Restauração "CHARLIE" clinicamente variável - Fonte: Própria. Este estudo obedeceu à Resolução 196/96

Esta avaliação ocorreu no consultório da Fundação com o auxílio de iluminação artificial, durante um período de três meses. Foram utilizados espelhos bucais e sondas exploradoras (SS White, Rio de Janeiro, RJ, Brasil), além de gazes descartáveis estéreis (SS Plus do Brasil Ltda, Maringá, PR, Brasil) para secagem das superfícies das restaurações e melhor visualização dessas.

Os instrumentos de pesquisa compreenderam um questionário para sondagem preliminar direcionado aos responsáveis, com intuito de delinear sua condição dietética, socioeconômica e a atenção dada à saúde bucal. Confeccionou-se uma ficha clínica contendo informações sociodemográficas, odontograma, índice CPO-D (Dentes Cariados, Perdidos e Obturados) e ficha de avaliação clínica das restaurações. Previamente à coleta de dados, os examinadores testaram o instrumento de pesquisa por meio do estudo piloto.

Os dados foram avaliados através de técnicas estatísticas descritivas, como média, intervalo de confiança, desvio padrão, valor mínimo e máximo, obtendo-se a análise inferencial por meio do cálculo individual e comparativo, sendo realizada a análise de variância oneway ANOVA e teste (T) para amostras independentes $(p<0,05)$. Os dados foram digitados e analisados no SPSS (Statistical Packag for Social Sciences) na versão 13.0. A margem de erro adotada para os testes estatísticos foi de 5\%.

\section{Resultados}

Constatou-se, nesta pesquisa, que a média de idade das crianças foi de 8,03 anos com intervalo de confiança de 7,58 a 8,48. Em relação à dieta fornecida às crianças, foi verificado que a adoção de frutas como sobremesa é priorizada nas três refeições diárias na Fundação. Entretanto, a higienização era realizada apenas após o almoço, sob supervisão de auxiliares de serviços gerais, os quais não possuíam treinamento adequado para executar tal atividade.
Quanto às condições socioeconômicas dos responsáveis, a maioria $(76,6 \%)$ possuia até o primeiro grau completo (Fig. 2).

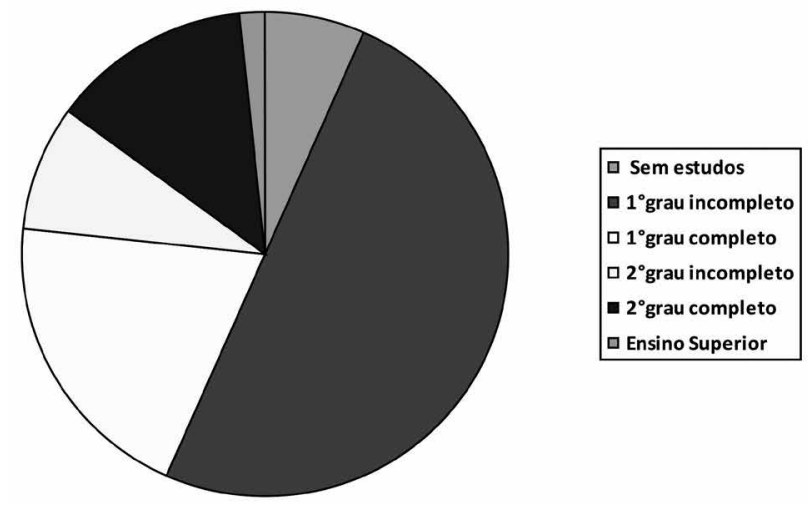

Figura 2 - Grau de escolaridade dos responsáveis pelas crianças

A renda familiar de até dois salários mínimos foi observada em 98,3\% dos casos. Em se tratanto da atenção direcionada à saúde bucal, foi constatado que a grande maioria já foi ao dentista alguma vez durante a vida (Fig. 3), dentre os quais, $57 \%$ se consultaram há menos de 1 ano. De todos os sujeitos de pesquisa, $93 \%$ sentiam necessidade de frequentar o consultório odontológico no momento da aplicação do questionário.

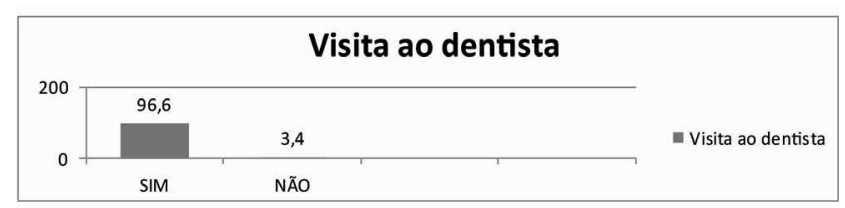

Figura 3 - Visita ao dentista dos responsáveis pelas crianças

Durante exame clínico preliminar, observou-se que a condição de saúde bucal da maioria das crianças apresentou-se precária, com atividade de cárie em $81 \%$ delas. Foram identificados elementos dentários com diferentes graus de comprometimento, infiltrações marginais, perda precoce, cáries ativas e inativas. Foi obtido o valor de 4,54 para o CPO-D médio dos escolares em estudo, observando-se que 
os do sexo masculino apresentaram um CPO-D mais elevado, não existindo, entretanto, diferença estatística significante $(P>0,05)$ comprovada pelo teste $t$ para amostras independentes (Tab. 2).

Tabela 2 - Média de CPO-D por gênero

\begin{tabular}{|c|c|c|c|c|}
\hline & Gênero & & & Estatística \\
\hline \multirow{8}{*}{ CPO_D } & \multirow{4}{*}{ Masculino } & \multicolumn{2}{|l|}{ Média } & 4,89 \\
\hline & & \multirow[t]{2}{*}{$\begin{array}{l}\text { Intervalo de confiança para a média com } \\
95 \% \text { de confiança. }\end{array}$} & Limite inferior & 3,72 \\
\hline & & & Limite superior & 6,05 \\
\hline & & \multicolumn{2}{|l|}{$\begin{array}{l}\text { Desvio Padrão } \\
\text { Minimo } \\
\text { Maximo }\end{array}$} & $\begin{array}{r}3,385 \\
0 \\
12 \\
\end{array}$ \\
\hline & \multirow{4}{*}{ Feminino } & \multicolumn{2}{|l|}{ Média } & 3,68 \\
\hline & & \multirow[t]{2}{*}{$\begin{array}{l}\text { Intervalo de confiança para a média com } \\
95 \% \text { de confiança. }\end{array}$} & Limite inferior & 2,76 \\
\hline & & & Limite superior & 4,60 \\
\hline & & \multicolumn{2}{|l|}{$\begin{array}{l}\text { Destio Padrão } \\
\text { Mínimo } \\
\text { Maximo }\end{array}$} & $\begin{array}{r}2,231 \\
0 \\
9\end{array}$ \\
\hline
\end{tabular}

Essa média de CPO-D mais elevada esteve nas crianças cujos pais ou responsáveis possuíam grau de escolaridade mais elevado (Ensino Médio Completo), apontando indícios de que o CPO-D das crianças não esteve relacionado com nível de esco- laridade desses, tal como foi verificado estatisticamente $(p>0,05)$. Analisando a renda familiar, constatou-se que as crianças com maior CPO-D foram aquelas cujos pais ou responsáveis ganham de um a dois salários mínimos $(p<0,05)$ (Tab. 3).

Tabela 3 - Média do CPO-D segundo cada rendimento dos pais ou responsáveis

\begin{tabular}{|c|c|c|c|c|}
\hline & \multicolumn{3}{|l|}{ Rendimento } & Estatística \\
\hline \multirow{8}{*}{ CPO_D } & \multirow{4}{*}{$\begin{array}{l}\text { Menos de } 1 \\
\text { Salários } \\
\text { Mínimos }\end{array}$} & \multicolumn{2}{|l|}{ Média } & 3,26 \\
\hline & & \multirow[t]{2}{*}{$\begin{array}{l}\text { Intervalo de confiança para a } \\
\text { média com } 95 \% \text { de confiança. }\end{array}$} & Limite inferior & 2,40 \\
\hline & & & Limite superior & 4,12 \\
\hline & & \multicolumn{2}{|l|}{$\begin{array}{l}\text { Desvio Padrão } \\
\text { Mínimo } \\
\text { Máximo }\end{array}$} & $\begin{array}{r}2,177 \\
0 \\
8 \\
\end{array}$ \\
\hline & \multirow{4}{*}{$\begin{array}{l}\text { de } 1 \text { a } 2 \\
\text { Salários } \\
\text { mínimos }\end{array}$} & \multicolumn{2}{|l|}{ Média } & 5,34 \\
\hline & & \multirow[t]{2}{*}{$\begin{array}{l}\text { intervalo de confiança para a } \\
\text { média com } 95 \% \text { de confiança. }\end{array}$} & Limite inferior & 4,14 \\
\hline & & & Limite superior & 6,54 \\
\hline & & \multicolumn{2}{|l|}{$\begin{array}{l}\text { Desvio Padrão } \\
\text { Mínimo } \\
\text { Máximo }\end{array}$} & $\begin{array}{r}3,327 \\
0 \\
12\end{array}$ \\
\hline
\end{tabular}


Constatou-se ainda que, o tempo de visita ao dentista não influenciou no CPO-D de seus filhos.

Após a coleta e análise descritiva dos dados, em relação às restaurações avaliadas, observou-se que a maior parte dos procedimentos foi realizada em pacientes do sexo masculino $(61,5 \%)$ e, principalmente, em alunos que faziam parte do $4^{\circ}$ ano letivo $(51,4 \%)$. Em relação ao elemento dentário mais frequentemente tratado, notou-se uma maior predominância de restaurações realizadas no elemento $46(16,5 \%)$.

Ao se avaliar as restaurações, após o período de três meses, foi observado que entre as 109 restaurações, houve uma perda de 17 dessas, correspondendo aos dentes decíduos que esfoliaram nesse intervalo. De acordo com os critérios da USPHS modificados, houve uma maior frequência de restaurações classificadas em condições satisfatórias, perfazendo um total de $59,8 \%$, dos quais $40,2 \%$ corresponderam a restaurações classificadas como ALFA, sem alterações clínicas, e 19,6\% consideradas como BRAVO, com alterações clinicamente aceitáveis. Foi obtido o valor de $40,2 \%$ para restaurações consideradas clinicamente inviáveis, sendo $26,1 \%$ classificadas como CHARLIE e $14,1 \%$ como DELTA.

\section{Discussão}

Os resultados deste trabalho negaram a hipótese de que o tratamento restaurador atraumático não influencia significativamente no desempenho clínico das restaurações e no tratamento da doença cárie. A Fundação Sementes de Vida beneficia 98 crianças carentes com idade de 5 a 13 anos, proporcionando a promoção social dessas. $\mathrm{O}$ tratamento restaurador atraumático apresentou maior indicação para os dentes decíduos que para os permanentes ${ }^{16}$. Logo, a fase de dentição mista, na qual as crianças da Fundação se encontravam, favoreceu a aplicabilidade desse tipo de tratamento.

Há evidências substanciais relacionando hábitos alimentares com a etiologia da cárie dental ${ }^{17}$, devido à relação entre a condição dentária, a função mastigatória e a ingestão de alimentos, principalmente açúcares ${ }^{18}$. Contudo, a quantidade de açúcar ingerida pelas crianças durante o período em que têm permanecido na fundação foi relativamente baixa, o que não justificou o alto índice de cárie diagnosticado. Entretanto, verificou-se que os hábitos de higiene bucal adotados pela instituição estavam distantes dos ideais, situação que pode ser considerada fator predisponente para a alta incidência de cárie registrada.

A odontologia tradicional estritamente curativista não consegue estabelecer melhores índices de saúde bucal, pois além da prevalência elevada de cárie dentária, encontram-se as barreiras econômicas por parte do paciente ${ }^{19}$. A renda familiar é o fator socioeconômico de maior importância em relação aos demais agentes determinantes do processo carioso ${ }^{20}$. As condições econômicas e sociais nas quais as crianças da fundação se encontram devem ser consideradas de risco, visto que a maioria dos responsáveis apresenta baixo grau de escolaridade e renda familiar insuficiente.

A incorporação dos pais no processo de determinação da saúde, através de orientações acerca da alimentação e higienização oral, se fez importante, concordando com Alves et al. ${ }^{21}$ (2004), devido à co-responsabilidade desses na promoção e manutenção das condições de saúde bucal de seus filhos.

De acordo com o Ministério da Saúde ${ }^{22}$, os valores do índice CPO-D correspondem aos seguintes graus de severidade: muito baixo $(0,0$ a 1,1$)$, baixo $(1,2$ a 2,6$)$, moderado $(2,7$ a 4,4$)$, alto $(4,5$ a 6,5$)$ e muito alto (6,6 e mais). Segundo o SB Brasil 2010, a prevalência de cárie nas crianças de 12 anos em nosso país foi $2,10^{23}$. O valor mensurado para as crianças da Fundação Sementes de Vida se classificou como alto, na medida em que possui CPO-D médio de 4,54, discordando dos dados obtidos nesse levantamento sobre saúde bucal da população brasileira. Valores elevados indicaram más condições de saúde bucal, frequentemente associadas às condições socioeconômicas desfavoráveis, dificuldade de acesso aos serviços e hábitos deletérios, como alto consumo de açúcar.

O TRA tem sido bem aceito pelas crianças e adultos em comparação com a abordagem tradicional ${ }^{24}$, devido à ausência da broca e da necessidade de administrar anestesia local. A maioria das crianças tratadas demonstrou-se satisfeita com o tratamento, independentemente da idade, não apresentando diferenças entre as respostas apresentadas pelos grupos ${ }^{2}$.

Alguns estudos têm questionado se o procedimento de selamento marginal é realmente capaz de evitar ou parar a lesão cariosa. Esse desfecho deve prevalecer especialmente para materiais bioativos, tais como ionômeros de vidro, que exercem um papel que não está limitado à vedação $0^{25,26}$.

A maioria das cáries oclusais não cavitadas restauradas com a técnica ART, colocadas em condições favoráveis, não avançam para a cavitação por um período de 6 anos, o que pode impedir que o dente seja inserido no ciclo de troca de restaurações, ao longo da vida ${ }^{27}$. Entretanto, o problema cárie dental ainda persiste no Brasil, exigindo a implantação de outras medidas pertinentes e que legitimem o papel dos órgãos públicos como protetor da saúde pública, proporcionando aos cidadãos em estado de vulneração dispor de ações, além do investimento nos serviços odontológicos, conforme as suas necessidades ${ }^{28}$. 


\section{Considerações finais}

A maioria das restaurações de cavidade classe I com cimento de ionômero de vidro de acordo com o critério USPHS apresentou sucesso clínico, comprovando a efetividade do TRA no tratamento da doença cárie. Confirmou-se também que a orientação direcionada à higienização bucal foi efetiva. Além disso, verificou-se que índice CPO-D médio geral das crianças assistidas pela Fundação Sementes de Vida estava acima da média nacional aferida para crianças de doze anos, constatando-se uma relação entre a baixa renda familiar e o CPO-D dessas.

\section{Agradecimentos}

Ao CNPq pela bolsa de iniciação científica, concedida ao primeiro autor através do Programa Institucional de Bolsa CNPq/PIBIC/UEPB.

\section{Albstract}

Atraumatic Restorative Treatment (ART) was developed to facilitate access to dental care for less affluent populations; it consisted of the partial removal of carious tissue keeping the affected dentin, using only hand instruments and restoring the cavity with a biomaterial, glass ionomer cement. Objective: To clinically assess Class I restorations with glass ionomer cement in children assisted by the Fundação Sementes da Vida (Seeds of Life Foundation), Campina Grande - PB, Brazil. Materials and Methods: An interventional, prospective study with quali-quantitative method was carried out. Clinical examination and restorations were performed in 85 infants, who presented occlusal caries in posterior teeth with diameter greater than $3 \mathrm{~mm}$ involving dentin. After three months, restorations were assessed according to the criteria of the modified U.S. Public Health Service (USPHS), and results were analyzed using descriptive statistical techniques with one-way ANOVA, and (T) test for independent samples $(P<0.05)$. Results: For all variables, except for the presence of secondary caries where the CHARLIE (C) score prevailed, the assessment indicated results consistent with the ALFA $(A)$ and BRAVO (B) scores, representing a significant degree of procedural success. Conclusions: Considering the assessment performed, it was found that the ART had a satisfactory performance because of the improvement on the clinical condition presented by children at the end of the study.

Keywords: Dental Atraumatic Restorative Treatment. Dental Caries. Glass lonomer Cements.

\section{Referências}

1. Melo MMDC, Souza WV, Lima MLC, Braga C. Fatores associados à cárie dentária em pré-escolares do Recife, Pernambuco, Brasil. Cad Saúde Pública 2011; 27(3):471-85.

2. Gibilini C, Ramalho DF, Sousa MLR. Avaliação da autopercepção do tratamento restaurador atraumático pelo paciente infantil. Rev Fac Odontol Passo Fundo 2011; 16(1):18-24.

3. Carvalho LM, Aldrigui JM, Bonifácio CC, Imparato JCP, Raggio DP. ART em cavidades atípicas. RGO 2009; 57(3):357-62.

4. Garbin CAS, Sundfeld RH, Santos KT, Cardoso JD. Aspectos atuais do tratamento restaurador atraumático. Rev Fac Odontol Passo Fundo 2008; 13(1):25-9.

5. Cantero-Fuentealba C, Bustos-Leal A, Cartes-Velásquez R, Ruiz-Medina O, Frencken JE. Evaluación de la capacitación en tratamiento restaurador atraumático, Facultad de Odontología, Universidad de Concepción, 2007 - 2008. Int J Odontostomat 2010; 4(2):139-47.

6. França TRT, Sedycias M, Silva RJ, Beatrice LCS, Vicente da Silva CH. Emprego do Cimento de Ionômero de Vidro: Uma Revisão Sistemática. Pesq Bras Odontoped Clin Integr 2010; 10(2):301-7.

7. American dental association. Guideline on Pediatric Restorative Dentistry. 2013/14;35(6):226-34.

8. Silva MAM, Mendes CAJ. O Tratamento Restaurador Atraumático em Saúde Pública e o custo dos materiais preconizados. Rev APS 2009; 12(3):350-6.

9. Frencken JE, Pilot T, Songpaisan Y, Phantumvanit P. Atraumatic restorative treatment (ART): rationale, technique and development. J Public Health Dent 1996; 56:135-40.

10. Tyas MJ. Avaliação clínica de restaurações de cimento de ionômero de vidro. J Appl Oral Sci 2006; 14(spe):10-3.

11. Lo ECM, Holmgren CJ, Hu D, Helderman WP. Six-year follow up of atraumatic restorative treatment restorations placed in Chinese school children. Community Dent Oral Epidemiol 2007; 35(5):387-92.

12. Frencken JE, Holmgren CJ. Tratamento Restaurador Atraumático para a Cárie Dentária. São Paulo: Santos Editora, 2001.

13. Cefaly DFG, Barata TJE, Tapety CMC, Bresciani E, Navarro MFL. Clinical evaluation of multisurface ART restorations. J Appl Oral Sci 2005; 13(1):15-9.

14. Cvar JR, Ryge G. Criteria for the clinical evaluation of dental materials. US Departament of Health Education and Welfare $1971 ; 790: 244$.

15. Santiago SL, Passos VF, Vieira AHM, Navarro MFL, Lauris JRP, Franco EB. Two-year clinical evaluation of resinous restorative systems in non-carious cervical lesions. Braz Dent J 2010; 21(3):229-34.

16. Wambier DS, Paganini F, Locatelli FA. Tratamento Restaurador Atraumático (Estudo da sua Aplicabilidade em Escolares de Tangará - SC). Pesq Bras Odontoped Clin Integr 2003; $3(2): 9-13$.

17. Johansson I, Birkhed D. A dieta e o processo cariogênico. In: Thylstrup A, Fejerskov O. Cariologia Clínica São Paulo: Livraria Santos Editora. 1995. p. 283-310. 
18. Krall E, Hayes C, Garcia R. How dentitions status and masticatory function affect nutrient intake. J Am Dent Assoc 1998; 129(9):1261-9.

19. Lima DC, Saliba NA, Moimaz SAS. Tratamento restaurador atraumático e sua utilização em saúde pública. Rev Gaúcha Odontol 2008; 56 (1):75-9.

20. Peres KGA, Bastos JRM, Latorre MRDO. Severidade de cárie em crianças e relação com aspectos sociais e comportamentais. Rev Saúde Públ 2000; 34:402-8.

21. Alves UM, Volschan BCG, Haas NAT. Educação em Saúde Bucal: Sensibilização dos pais de crianças atendidas na clínica integrada de duas universidades privadas. Pesq Bras Odontoped Clin Integr 2004; 4(1):47-51.

22. Brasil. Ministério da Saúde. Programa de Saúde Bucal: Levantamento Epidemiológico em Saúde Bucal - Cárie Dental. Brasília: Ministério da Saúde, 1996.

23. Brasil. Ministério da Saúde (MS). Secretaria de Vigilância em Saúde. Secretaria de Assistência à Saúde. Departamento de Atenção Básica. Coordenação Nacional de Saúde Bucal. Pesquisa Nacional de Saúde Bucal -2010. Nota para a imprensa. Brasília: MS; 2010.

24. Schriks MCM, Van Amerongen WE. Atraumatic perspective of ART: Psychological and physiological aspects of treatment with and without rotary instruments. Community Dent Oral Epidemiol 2003; 31:15-20.

25. Tyas MJ, Anusavice KJ, Frencken JE, Mount GJ. Minimal intervention dentistry - a review. Int Dent J 2000; 50:1-12.

26. Mejàre I, Lingström P, Petersson LG, Holm AK, Twetman S, Källesta $\mathrm{C}$ et al. Caries-preventive effect of fissure sealants: a systematic review. Acta Odontol Scand 2003; 61:321-30.

27. Frencken JE, Wolke J. Clinical and SEM assessment of ART high-viscosity glass-ionomer sealants after 8-13 years in 4 teeth. J Dent 2010; 38:59-64.

28. Costa SM, Abreu MHNG, Vasconcelos M, Lima RCGS, Verdi M, Ferreira EF. Desigualdades na distribuição da cárie dentária no Brasil: uma abordagem bioética. Ciênc saúde coletiva $2013 ; 18(2): 461-70$.

Endereço para correspondência:

Ana Isabella Arruda Meira Ribeiro

BR 104, KM 119, S/N, Condomínio Nações Residence

Privê, Quadra F, Lote 11

Sítio Guabiraba

58117-000 Lagoa Seca / PB

Fone: 8333373649 / 87900118

E-mail: isaro_jesus@hotmail.com

Recebido: 11/11/2013. Aceito: 11/05/2014. 